\title{
CRITICAL SUBMANIFOLDS OF DIFFERENTIABLE MAPPINGS. II
}

\author{
BY SAMIR KHABBAZ AND EVERETT PITCHER
}

Communicated by E. Pitcher, September 15, 1966

In this paper we discuss two more aspects of a general problem described amply in [4], a paper whose various conventions we continue to use. In particular manifolds and submanifolds are to be taken in the differentiable sense, and a differentiable mapping $f: M^{m} \rightarrow R^{r}$ from an $m$-dimensional manifold with boundary into $r$-dimensional euclidean space is called ordinary if its rank at each point is the maximum possible.

1. Factoring through immersion. The following Lemma will be used in the proof of the next theorem.

LEMMA: Let $f: R^{n} \rightarrow R^{n}$ be a $C^{1}$ function such that the following three conditions hold.

(1) $f\left(x_{1}, \cdots, x_{n}\right)=\left(y_{1}\left(x_{1}, \cdots, x_{n}\right), \cdots, y_{n}\left(x_{1}, \cdots, x_{n}\right)\right)$,

(2) $f(0, \cdots, 0)=(0, \cdots, 0)$,

(3) setting $K^{*}=\left\{\left(x_{1}, \cdots, x_{n}\right) \in R^{n} \mid x_{n}=0\right\}$ and $R_{-}=\left\{\left(y_{1}, \cdots, y_{n}\right)\right.$ $\left.\in R^{n} \mid y_{n} \leqq 0\right\}$, we have $f \mid\left(R^{n}-K^{*}\right)$ is ordinary, $f \mid K^{*}$ is an embedding and $f\left(R^{n}\right) \subset R_{-}$.

Then for any point $x_{+}$in $R(>)=\left\{\left(x_{1}, \cdots, x_{n}\right) \in R^{n} \mid x_{n}>0\right\}$ and for any point $x_{-}$in $R(<)=\left\{\left(x_{1}, \cdots, x_{n}\right) \in R^{n} \mid x_{n}<0\right\}$ we have

$$
\mathrm{J} f\left(x_{+}\right) \cdot \mathrm{J} f\left(x_{-}\right)<0 .
$$

Proof. The hypothesis implies that the hyperplane $P$ $=\left\{\left(y_{1}, \cdots, y_{n}\right) \in R^{n} \mid y_{n}=0\right\}$ is tangent to $f\left(K^{*}\right)$ at $(0)=(0, \cdots, 0)$, hence expanding $\mathrm{J} f(x)$, the determinant of the Jacobian matrix of $f$, near (0) in terms of the last row we obtain $\mathrm{J} f(x)=A\left(x_{1}, \cdots, x_{n}\right)$ $+B\left(x_{1}, \cdots, x_{n}\right)\left(\partial y_{n} / \partial x_{n}\right)$ where $A$ is the sum of the first $(n-1)$ terms and $B(0, \cdots, 0) \neq 0$. Since $f \mid\left(R^{n}-K^{*}\right)$ is ordinary it is suffcient to prove the Lemma for some $x_{+}$in $R(>)$ and some $x_{-}$in $R(<)$. Deny this. Then for $x_{n} \neq 0, \mathrm{~J} f(x)$ is either always positive or always negative, and we may assume for definiteness that $B(0, \cdots, 0)>0$ and $\mathrm{J} f(x)>0$ for all $x=\left(x_{1}, \cdots, x_{n}\right), x_{n} \neq 0$. (For the case $\mathrm{J} f>0$ and $B(0)<0$ see the note below.) Further we may assume by continuity that on some neighbourhood $C$ of $(0)$, say $C=\left\{x \in R^{n} \mid\|x\| \leqq a\right\}$, $B$ is positive. Also we may assume that $f\left(C_{a}\right)$, where $C_{a}$ $=\left\{x \in R^{n} \mid\|x\|=a\right\}$, is at a positive distance $d$ from $P$. This may be accomplished for instance by composing $f$ with an appropriate dif- 
feomorphism of $R^{n}\left(y_{1}, \cdots, y_{n}\right)$ to yield another mapping having the same properties as $f$ plus this latter property. Next, let $m: R \rightarrow R$ be a $C^{\infty}$ function such that: (a1) $\mid m(t)<d / 2$, (a2) $m(0)=0$, (a3) $m^{\prime}(0)>0$, and (a4) $m^{\prime}(t) \geqq 0$. (Note: If $\mathrm{J} f>0$ and $B<0$ use $-m(t)$.) Now consider the following alteration $g$ of $f$ given by $g\left(x_{1}, \cdots, x_{n}\right)$ $=\left(y_{1}\left(x_{1}, \cdots, x_{n}\right), \cdots, y_{n-1}\left(x_{1}, \cdots, x_{n}\right), y_{n}\left(x_{1}, \cdots, x_{n}\right)+m\left(x_{n}\right)\right)$. Then $\mathrm{J} g(x)=\mathrm{J} f(x)+B\left(x_{1}, \cdots, x_{n}\right) m^{\prime}\left(x_{n}\right)$, and $g \mid C$ has the following properties: (b1) $\mid g(x)-f(x) \|<d / 2$ for any $x$ on $C_{a}$, (b2) $g \mid\left(C \cap K^{*}\right)$ $=f \mid\left(C \cap K^{*}\right)$, (b3) $\mathrm{J} g(0) \neq 0$, and (b4) $\mathrm{J} g(x)>0$ for $x$ in $C-K^{*}$. Each (bi) follows from the corresponding (ai).

Finally consider the composite map $\left(p_{n} g\right) \mid C$ where $p_{n}$ is the projection map $p_{n}\left(y_{1}, \cdots, y_{n}\right)=y_{n}$. From (b3) it follows that maximum $p_{n} g \mid C$ is greater than zero. From (b1) it follows that this maximum is not attained on $C_{a}$; and from (b2) it follows that this maximum is not attained on $K^{*} \cap C$. From (b4) it also follows that this maximum is not attained on the open set $C-\left(K^{*} \cap C_{a}\right)$ either. This contradiction establishes the Lemma.

THEOREM 1. Let $N$ be a compact n-dimensional manifold, and let $K$ be a compact $(n-1)$-dimensional submanifold of $N$. Assume further that: (1) There exists a differentiable mapping $f: N \rightarrow R^{n}$ such that both $f \mid(N-K)$ and $f \mid K$ are immersions (i.e. ordinary) and (2) at least one of the following three conditions (a), (b), and (c) is satisfied: (a) Both $K$ and $N$ are orientable, (b) $N$ is orientable and $K$ is connected, (c) each component of $K$ is simply connected. Then $f$ can be factored into an immersion of $N$ into $R^{n+1}$ followed by a projection of $R^{n+1}$ onto $R^{n}$.

Proof. We first show that the normal bundle $V$ of $K$ in $N$ is a product bundle. This is well known for cases (a) and (c), and is readily verified. Deny this is so in case (b), then since $K$ is connected, it is not hard to see that $N-K$ must be connected. Now let $P$ be a plane of support of $f(N)$ in $R^{n}$, let $y$ be a point of contact, and let $x$ be a point of $N$ (necessarily in $K$ ) such that $f(x)=y$. Next let $\left(y_{1}, \cdots, y_{n}\right)$ be a coordinate system in $R^{n}$ about $y$, and such that: $y=(0, \cdots, 0), P$ is the plane $\left(y_{1}, \cdots, y_{n-1}, 0\right)$, and the line $\left(0, \cdots, 0, y_{n}\right)$ is perpendicular to $P$. By (1) of the theorem we may choose a coordinate system $\left(x_{1}, \cdots, x_{n}\right)$ in a neighbourhood $E$ of $x$ in $N$ such that $x=(0, \cdots, 0), K^{*}=K \cap E$ corresponds to the points $\left(x_{1}, \cdots, x_{n-1}, 0\right)$, and $f \mid K^{*}$ is an embedding. Then using the same notation as in the Lemma, the hypotheses of the Lemma are satisfied and hence its conclusion holds. This together with the facts that $N-K$ is connected, and that $f \mid(N-K)$ is ordinary are easily seen to 
imply that $N$ is not orientable. This contradicts (b) and implies that $V$ is a product bundle. (Actually, assuming $N$ in (1) is orientableimplies $K$ has an orientable component.) Hence in all cases, $K$ has a neighbourhood $F$ in $N$ of the form $K \times R$, in which $K$ corresponds to the set $K \times 0$. Now let $h: R \rightarrow R$ be a $C^{\infty}$ function such that $h^{\prime}(0) \neq 0$ and $h(t)=0$ for all $t$ with $|t|>1$. Now define a function $H: N \rightarrow R^{n+1}$ by $H(z, t)=(f(z, t), h(t))$ for $(z, t)$ in $K \times R=F$, and for $x$ in $N-F$ define $H$ by $H(x)=(f(x), 0)$. The newly defined map $H$ is the required immersion, while the projection is the obvious one.

(For a good discussion of factoring maps through immersions when $n=2$, the reader is referred to $[2]$.)

Corollary. If $N$ is orientable in Theorem 1 , then $w(N)=1$ and $w(K)=1$, so that in particular both $N$ and $K$ have even Euler characteristics.

2. Relationship with vector fields. Next we recall that if the standard $n$-sphere $S^{n}$ is projected on to the hyperplane $R^{r}$ through the origin, $n \geqq r$, and if $p$ denotes this projection, then the set of critical points of $p$ is $S^{r-1}$ and $p \mid S^{r-1}$ is an embedding. In the following theorem the analogous situation is investigated for an arbitrary manifold.

In this section let $N$ denote a connected $n$-dimensional manifold with boundary (perhaps empty) and let $N_{0}$ denote $N$ with a point deleted.

Theorem 2. Let $K$ be an $(r-1)$-dimensional compact submanifold with boundary of $N, n \geqq r$, and suppose that there exists a differentiable mapping $f: N \rightarrow R^{r}$ such that: (A) $f \mid(N-K)$ and $f \mid K$ are ordinary, and (B) $f(K)$ is a submanifold of $R^{r}$. Then $N_{0}$ admits $r-1$ linearly independent vector fields.

Proof. Let $f(K)$ be the union of its components $F_{1}, \cdots, F_{s}$. Let $y_{i}$ be a point of $F_{i}$, and set $g=f \mid K$. Then since $g$ is an immersion $g^{-1}\left(y_{i}\right)$ is finite consisting say of the points $x_{i}^{1}, \cdots, x_{i}^{t_{i}}$. For each $x_{i}^{j}$ let $D_{i}^{j}$ be a closed $n$-disc in $N$ containing $x_{i}^{j}$ in its interior and such that the $D_{i}^{y \prime}$ 's are disjoint. (Note: If $x$ is a boundary point of $N$ replace "disc" by "half-disc".) Now about each $y_{i}$ let $D_{i}$ be a closed disk in $F_{i}$, sufficiently small so that $g^{-1}\left(D_{i}\right) \subset \cup_{j} D_{j}^{i}$. Finally let $L$ denote $f(K)$ with the interior of each $D_{i}$ removed, and let $M$ denote $N$ with the interior of each $D_{i}^{j}$ removed.

Since $f(K)$ is an $(r-1)$-dimensional submanifold of $R^{r}$, it follows from Alexander duality that $\tau\left(R^{r}\right)$ admits a nonvanishing field of vectors normal to $f(K)$ with respect to some Riemannian metric on $R^{r}$. This field induces a cross section $c$ of $\tau\left(R^{r}\right) \mid L$. Now the only 
obstruction to extending $c$ to a nonvanishing cross section $v$ defined on $R^{r}$ is an element of $H^{r}\left(R^{r}, \mathrm{~L} ; \pi_{r-1}\left(S^{r-1}\right)\right)=0$. Thus $v$ determines a line subbundle $V$ of $\tau\left(R^{r}\right)$, and using the Riemannian metric on $R^{r}$ we may write $\tau\left(R^{r}\right)=V \oplus W$ where $W$ is an $(r-1)$-plane subbundle of $\tau\left(R^{r}\right)$. Next consider the composite mapping $h$ given by $\tau(M) \rightarrow \tau\left(R^{r}\right)$ $\rightarrow W$, where the first mapping is given by $d f$ the differential of $f$, and the second is the projection induced by the Whitney sum decomposition. Note now that (A) of the proposition together with the way in which we constructed $L$ and $M$ imply that $h$ maps fibers linearly on to fibers. Hence $\tau(M)$ decomposes into the Whitney sum $\tau_{n-r+1} \oplus \tau_{r-1}$ where $\tau_{r-1}$ is an $(r-1)$-plane bundle over $M$ induced from $W$ by $f$. Since $R^{r}$ is contractible $W$ is trivial and hence so is $\tau_{r-1}$. Thus $M$ admits $r-1$ linearly independent vector fields. Finally, since the $D_{i}^{\text {j's }}$ are closed and disjoint they may all be gathered inside one disc of $N$, and the theorem follows.

Corollary. Let $K$ be an $(n-1)$-dimensional compact submanifold of the real $n$-dimensional projective space $P^{n}, n>8$. Then there exists no differentiable mapping $f: P^{n} \rightarrow R^{n}$ subject to: (A) $f \mid\left(P^{n}-K\right)$ and $f \mid K$ are immersions and (B) $f(K)$ is a submanifold of $R^{n}$.

Proof. By Theorem 2, the existence of an $f$ implies the existence of $n-1$ linearly independent vector fields over $P_{0}^{n}$. Using Sanderson's lemma [1, p. 332], it suffices to prove either that $P^{n}$ does not admit $n-1$ linearly independent vector fields over $P^{n-1} \subset P_{0}^{n}$, or that $P^{n-1}$ is not immersible in $R^{n}$. Then the Corollary follows from well known facts concerning immersions of $P^{m}$. A proof can for instance be given as follows: For $8<n \leqq 2^{4}-1$ see [3]; for $n \neq 2^{r}-1, n \neq 2^{r}, r \geqq 4$ the result follows from examining the Striefel-Whitney classes; for $n=2^{r}$ $-1, r \geqq 4\left[1\right.$, Theorem 9.5, p. 331] applies since $P_{0}^{2^{r}-1}$ contains $P^{2^{r^{-1}}+2}$; and for $n=2^{r}, r \geqq 4$ the result follows from [5].

REMARKs. The above results and those in [4] are by no means complete or best possible, and should be regarded merely as being typical. There is however a worth while generalization of Theorem 2 which we shall discuss since it allows $f \mid K$ to have additional self-intersections.

THEOREM 2'. Suppose that $K$ is a $k$-dimensional compact submanifold with boundary of $N, n \geqq r$, and assume that there exists a differentiable function $f: N \rightarrow R^{r}$ such that: (A) $f \mid(N-K)$ and $f \mid K$ are ordinary, (B) in some triangulation $T$ of $R^{r}, f(K)$ is the underlying space of a subcomplex of $T$, and lastly (C) there exist $r-k$ linearly independent cross sections $c_{1}, \cdots, c_{r-k}$ of $\tau\left(R^{r}\right) \mid(f(K)-S$ ) (where $S$ is a finite subset of 
$f(K)$ possibly empty) such that for any $x$ in $K \cap f^{-1}(f(K)-S)$, if $T_{x}$ denotes the fiber of $\tau(K)$ over $x$ and if $d g: \tau(K) \rightarrow \tau\left(R^{r}\right)$ is the differential of $g=f \mid K$, then $c_{1}(f(x)), \cdots, c_{r-k}(f(x))$ and $d g\left(T_{x}\right)$ span the fiber of $\tau\left(R^{r}\right)$ over $f(x)$.

Then $N_{0}$ admits $k$ linearly independent vector fields.

Proof. For the $y_{i}$ 's of the proof of Theorem 2, take a finite set $Q$ containing $S$ and such that $H^{k}(f(k)-Q)=0$. Construct the $D_{i}^{\text {'s }}$ as before, and replace the $D_{i}$ 's by open stars of the $y_{i}{ }^{\prime}$ 's in some fine enough subdivision of $f(K)$. Define $L$ and $M$ as before. Then since the Stiefel manifold $V_{r, r-b}$ of $r-k$ frames in $r$-space is $k-1$ connected, and since $K$ is $k$-dimensional the only obstruction to extending $c_{1} \mid L$, $\cdots, c_{r-k} \mid L$ to independent cross sections $v_{1}, \cdots, v_{r-k}$ over $R^{r}$ is an element of $H^{k+1}\left(R^{r}, L ; \pi_{k}\left(V_{r, r-k}\right)\right)$ which is zero by our choice of the set $Q$. The $v_{i}$ 's define a subbundle $V$ of $\tau\left(R^{r}\right)$, and the rest is as before.

\section{BIBLIOGRAPHY}

1. P. Baum and W. Browder, The cohomology of quotients of classical groups, Topology 3 (1965), 305-336.

2. André Haefliger, Quelques remarques sur les applications différentiables d'une surface dans le plan, Ann. Inst. Fourier (Grenoble) 10 (1960), 47-60.

3. M. Hirsch, Data on immersions and embeddings of projective spaces. Seattle Conference on Differential and Algebraic Topology, Seattle, Washington, 1963.

4. S. Khabbaz and E. Pitcher, Critical submanifolds of differentiable mappings, Bull. Amer. Math. Soc. 73 (1967), 164-168.

5. I. M. James, On the immersion problem for real projective spaces, Bull. Amer, Math. Soc. 69 (1963) 231-238.

LEHIGH UNIVERSITY 